\title{
Altered Membrane Sodium Transport in Bartter's Syndrome
}

\author{
Jerry D. Gardner, Artemis P. Simopoulos, Allen Lapey, and \\ SHLOMo SHibolet \\ From the Digestive and Hereditary Diseases Branch and the Metabolic \\ Diseases Branch, National Institute of Arthritis and Metabolic Diseases; \\ and the Endocrinology Branch, National Heart and Lung Institute; \\ National Institutes of Health, Bethesda, Maryland 20014
}

\begin{abstract}
A B S T RACT To explore the possibility that Bartter's syndrome is the manifestation of an inherited abnormality of sodium transport, we have measured various parameters of sodium transport in erythrocytes from patients with Bartter's syndrome, their siblings, and their parents. Sodium transport in six of the eight patients with Bartter's syndrome differed significantly from that in the other two patients. On the basis of this difference, the patients were divided into two groups (type I and type II). In the six type I patients, fractional sodium outflux $(0.38 \pm 0.05 / \mathrm{hr}$ [SD] $)$ was significantly less than normal $(0.50 \pm 0.07)$ and erythrocyte sodium concentration $(9.48 \pm 0.84 \mathrm{mmoles} / \mathrm{liter}$ cells per $\mathrm{hr})$ was significantly greater than normal $(5.24 \pm 0.66)$. In the two type II patients, none of the measured parameters of sodium transport differed significantly from normal. Erythrocyte sodium transport in the relatives of three type I patients was altered in a way similar to that in the type I patients and was significantly different from that in the relatives of a type II patient. These findings indicate the presence of inherited alterations of erythrocyte sodium transport in certain patients with Bartter's syndrome:
\end{abstract}

\section{INTRODUCTION}

In 1962 Bartter, Pronove, Gill, and MacCardle (1) described a syndrome characterized by idiopathic juxtaglomerular hyperplasia, hyperaldosteronism, hypokalemia, elevated concentrations of plasma renin and angiotensin, normal blood pressure, and the absence of peripheral edema. The etiology of this syndrome is unknown; however, vascular insensitivity to angiotensin (1), autonomous overproduction of renin (2), and defective sodium

A preliminary report of this work was presented at the Annual Meeting of the American Society for Clinical Investigation, 4 May 1970.

Received for publication 28 October 1972 and in revised form 5 January 1972. reabsorption in the proximal renal tubule (3) have each been postulated to be the primary pathophysiologic event. Bartter's syndrome has been presumed to be a genetic disorder with an autosomal recessive pattern of inheritance (2) and has been reported to occur among siblings (4-6) and in one patient who was the product of a consanguineous union (6). To explore the possibility that Bartter's syndrome is a manifestation of a generalized, inherited abnormality of sodium transport, we have measured various parameters of sodium transport in erythrocytes from patients with Bartter's syndrome, their siblings and their parents.

\section{METHODS}

Fresh heparinized blood was obtained from 16 normal male and female human volunteers (ages 9-35), 8 patients with Bartter's syndrome (1), 8 parents, 8 siblings, 7 patients with secondary hyperaldosteronism from other causes $(7,8)$, and 1 patient with primary hyperaldosteronism (7) whose diagnosis was confirmed by subsequent surgical removal of an adrenal adenoma. Each of the patients with Bartter's syndrome demonstrated juxtaglomerular hyperplasia on renal biopsy, hypokalemia, elevated aldosterone secretory rate, increased plasma renin, normal blood pressure, the absence of peripheral edema, and a diminished vasopressor response to intravenous angiotensin infusion. Three of these patients with Bartter's syndrome have been the subjects of previous reports $(1,9,10)$.

The cells were separated by centrifugation at $3000 \mathrm{~g}$ for $5 \mathrm{~min}$ at room temperature and the plasma and buffy coat removed by aspiration. Erythrocytes were then washed three times with iced isosmotic $\mathrm{NaCl}$, divided into two portions, and placed in identical preincubation solutions (prewarmed to $37^{\circ} \mathrm{C}$ ) at approximately $10 \%$ hematocrit. ${ }^{24} \mathrm{Na}$ was added to the preincubation mixture containing erythrocytes which were subsequently used for sodium outflux determinations. After $2 \mathrm{hr}$ of incubation at $37^{\circ} \mathrm{C}$ in an oscillating water bath (American Instrument Co., Inc., Silver Springs, Md.), samples were taken for determination of hematocrit and hemoglobin concentration. The cells were then separated by centrifugation and washed three times with iced isosmotic choline chloride. A portion of the washed cells was hemolyzed and diluted for the determination of sodium and hemoglobin concentration. Hemoglobin was measured using the 
cyanmethemoglobin method (11). Sodium concentration was measured with an Instrumentation Laboratory model 143 flame photometer (Instrumentation Laboratory, Inc., Lexington, Mass.). Erythrocyte sodium concentration was calculated after the method of Sachs and Welt (12).

The standard preincubation and incubation solutions had the following composition (mM): $\mathrm{NaCl}, 146 ; \mathrm{KCl}, 6.0$; tris buffer $(\mathrm{pH}=7.4), 10$; glucose, 11.1. Whenever the concentration of sodium or potassium was decreased, an equimolar amount of choline was substituted. Ethacrynic acid, when added to the incubation medium, was present at a final concentration of $1 \mathrm{~mm}$.

Sodium outflux. The cells which were preincubated in the medium containing ${ }^{24} \mathrm{Na}$ were added to various incubation solutions (prewarmed to $37^{\circ} \mathrm{C}$ ) at an hematocrit of approximately $5 \%$. After mixing thoroughly, samples were taken at time "zero" and various intervals thereafter. The samples were chilled, the cells separated by centrifugation at $10,000 \mathrm{~g}$ for $15 \mathrm{sec}$, and a portion of the supernate was taken for measurement of radioactivity. At some time during the experiment, a portion of the incubation mixture (i.e., cells plus medium) was sampled. The cells were lysed and the protein was precipitated by adding $5 \%$ perchloric acid and centrifuging at $10,000 \mathrm{~g}$ for $30 \mathrm{sec}$. Appropriate control experiments demonstrated no significant entrapment of ${ }^{24} \mathrm{Na}$ in the precipitate. The supernate was added to a counting vial and its radioactivity measured. Initially samples were taken at $0,15,30,45$, and $60 \mathrm{~min}$; however, since the fractional sodium outflux was found to be constant over this period, fractional sodium outflux was calculated from samples taken at 0 and $30 \mathrm{~min}$. Radioactivity was determined with a Packard model 3320 liquid scintillation spectrometer (Packard Instrument Co., Inc., Downers Grove, Ill.).

Fractional sodium outflux $(k)$ was calculated from the following equation (13):

$$
k=\frac{-\ln \left(\mathrm{A}_{30} / \mathrm{A}_{0}\right)}{\mathrm{t}}
$$

where $k=$ fractional sodium outflux $\left(\mathrm{hr}^{-1}\right) ; \mathrm{A}_{0}$ and $\mathrm{A}_{30}=$ amount of radioactivity in a portion of cells at 0 and 30 $\min ; \mathrm{t}=$ times (hours).

Sodium influx. The cells which were preincubated in the nonradioactive medium were added to various prewarmed incubation solutions which contained ${ }^{24} \mathrm{Na}$ but were otherwise identical in composition to those used for outflux determination. The cells were mixed thoroughly and samples were taken at 0 and $30 \mathrm{~min}$. The samples were chilled and the cells washed four times by alternate centrifugation and resuspension in iced isosmotic choline chloride. The cells were lysed and the protein precipitated by adding $5 \%$ perchloric acid and centrifuging at $10,000 \mathrm{~g}$ for $30 \mathrm{sec}$. The supernate was added to a counting vial and its radioactivity measured. At some time during the experiment, a portion of the incubation mixture was taken for determination of hemoglobin concentration. The volume of cells counted was calculated from the hemoglobin concentration of the incubation mixture and the previously measured hemoglobin content per volume of cells. Changes in cell water (determined gravimetrically) during the $30-\mathrm{min}$ incubation period were negligible.

Sodium influx $\left(\mathrm{J}_{\mathrm{in}}\right)$ was calculated from the following equation (13):

$$
\mathrm{J}_{\mathrm{in}}=\left[\frac{k \mathrm{U}}{1-\mathrm{e}^{-k \mathrm{t}}}\right] / \mathrm{SA}
$$

where $\mathrm{J}_{\text {in }}=$ sodium influx (mmoles/liter cells per hour); $\mathrm{U}=$ uptake of radioactivity/liter of cells at time " $t$ "; $\mathrm{SA}=$ specific activity of the incubation medium; $t=$ time (hours).

Radioactivity was usually such that the standard deviation of the observed counts was $1 \%$ or less and all counts were corrected for decay. Liquid scintillation counting was performed using $20 \mathrm{ml}$ of solution composed of 15 parts toluene (J. T. Baker Chemical Co., Phillipsburg, N. J.), 5 parts Triton X-100 (New England Nuclear Corp., Boston, Mass.) and 1 part Liquifluor (New England Nuclear Corp.).

To compare the specific activity of the sodium leaving the cells with the specific activity of the sodium in the cells, erythrocytes were incubated for $2 \mathrm{hr}$ in the standard incubation medium containing ${ }^{24} \mathrm{Na}$. The cells were then washed four times with cold, isosmotic choline chloride and incubated in a sodium-free medium for $1 \mathrm{hr}$. At 15-min intervals, samples were taken, chilled, and the sodium concentration and radioactivity were measured in the cells and in the supernate.

To study the effect of plasma from patients with Bartter's syndrome on erythrocyte sodium transport, fresh plasma and erythrocytes were obtained from three normal subjects and from three patients with Bartter's syndrome. The sodium and potassium concentrations of the plasma were adjusted to 146 and $6 \mathrm{~mm}$, respectively. Erythrocytes from normal subjects and from patients with Bartter's syndrome were each preincubated for $3 \mathrm{hr}$ with ${ }^{24} \mathrm{Na}$ in normal plasma and in Bartter's plasma. Each portion of cells was then washed four times and incubated for $30 \mathrm{~min}$ in the same plasma used for its preincubation. Erythrocyte sodium concentration and fractional sodium outflux were determined as described previously.

To measure erythrocyte lactate production, washed red cells were incubated in the standard incubation medium (hematocrit approximately 25\%) and the appearance of lactate in the extracellular fluid was measured enzymatically $^{1}$ at 15 -min intervals. Initial studies demonstrated that lactate production by erythrocytes from patients with Bartter's syndrome and from normal subjects was constant for at least $3 \mathrm{hr}$. Lactate production was calculated from samples taken after 30 and $90 \mathrm{~min}$ of incubation using the following equation:

$$
\mathrm{P}_{\mathrm{L}}=\left[\frac{\mathrm{H}}{1.0-\mathrm{H}}\right]\left(\mathrm{L}_{90}-\mathrm{L}_{30}\right)
$$

where $P_{L}=$ lactate production (mmoles/liter cells per hour); $\mathbf{H}=$ hematocrit of the incubation mixture; $\mathrm{L}_{30}$, $\mathrm{L}_{90}=$ mmoles lactate/liter incubation mixture at 30 , and $90 \mathrm{~min}$ respectively.

Unless otherwise specified, statistical comparisons were performed using Students' $t$ test.

\section{RESULTS}

Table I summarizes the data for sodium transport in erythrocytes from patients with Bartter's syndrome, patients with secondary hyperaldosteronism from other causes, and one patient with primary hyperaldosteronism. Sodium transport in erythrocytes from six of the eight patients with Bartter's syndrome differed from that in the other two patients in terms of erythrocyte sodium concentration, fractional sodium outflux (in both the

\footnotetext{
${ }^{1}$ Boehringer and Soehne Co., Mannheim, Germany. From Calbiochem, San Diego, Calif.
} 
TABLE I

Sodium Transport in Erythrocytes from Patients with Bartter's Syndrome

\begin{tabular}{|c|c|c|c|c|c|c|c|}
\hline \multirow[b]{2}{*}{ Subjects } & \multirow{2}{*}{$\begin{array}{l}\text { Erythrocyte } \\
\text { sodium } \\
\text { concentration }\end{array}$} & \multicolumn{3}{|c|}{ Fractional sodium outflux } & \multicolumn{3}{|c|}{ Sodium influx } \\
\hline & & Control* & +ETA & Change§ & Control & +ETA & Change \\
\hline & $\begin{array}{l}\text { mmoles/ } \\
\text { liter cells }\end{array}$ & & $h r^{-1}$ & & & nmoles/liter cells & $h r$ \\
\hline \multicolumn{8}{|l|}{ Bartter's syndrome } \\
\hline C. J. (19 M) & 9.93 & 0.405 & 0.234 & -0.171 & 1.69 & 1.95 & 0.26 \\
\hline K. B. $(4 \mathrm{~F})$ & 10.99 & 0.343 & 0.208 & -0.135 & 1.52 & 2.08 & 0.56 \\
\hline J. S. $(2 \mathrm{~F})$ & 8.82 & 0.428 & 0.263 & -0.165 & 1.74 & 1.98 & 0.24 \\
\hline M. J. (12 F) & 8.86 & 0.300 & 0.162 & -0.138 & 1.63 & 1.90 & 0.27 \\
\hline D. C. $(13 \mathrm{~F})$ & 9.30 & 0.359 & 0.208 & -0.151 & 1.64 & 2.11 & 0.47 \\
\hline J. E. $(41 \mathrm{~F})$ & 8.99 & 0.432 & 0.253 & -0.179 & 1.73 & 1.96 & 0.23 \\
\hline Mean \pm 1 sD & $9.48 \pm 0.84 \|$ & $0.38 \pm 0.05 \|$ & $0.22 \pm 0.04 \|$ & $-0.16 \pm 0.03$ & $1.66 \pm 0.08$ & $2.00 \pm 0.08 \|$ & $0.34 \pm 0.14 \|$ \\
\hline J. A. $(37 \mathrm{~F})$ & 5.56 & 0.537 & 0.365 & -0.172 & 1.80 & 1.71 & -0.09 \\
\hline R. B. $(9 \mathrm{M})$ & 5.16 & 0.526 & 0.313 & -0.213 & 1.62 & 1.63 & . 0.01 \\
\hline Mean \pm 1 SD & $5.36 \pm 0.28$ & $0.53 \pm 0.01$ & $0.34 \pm 0.04$ & $-0.19 \pm 0.03$ & $1.71 \pm 0.13$ & $1.67 \pm 0.06$ & $-0.04 \pm 0.07$ \\
\hline Normal subjects (16) & $5.24 \pm 0.66$ & $0.50 \pm 0.07$ & $0.34 \pm 0.04$ & $-0.16 \pm 0.03$ & $1.74 \pm 0.20$ & $1.70 \pm 0.28$ & $-0.04 \pm 0.12$ \\
\hline Secondary hyperaldo. (7) & $5.52 \pm 0.73$ & $0.51 \pm 0.05$ & $0.33 \pm 0.02$ & $-0.18 \pm 0.05$ & $1.69 \pm 0.23$ & $1.71 \pm 0.26$ & $0.02 \pm 0.14$ \\
\hline Primary hyperaldo. (1) & 5.29 & 0.493 & 0.342 & -0.151 & 1.68 & 1.71 & 0.03 \\
\hline
\end{tabular}

The values for all subjects were determined in duplicate.

Age and sex of patients with Bartter's syndrome in parentheses.

* Control incubation medium (mM): $\mathrm{Na}, 146 ; \mathrm{K}, 6.0$.

$\ddagger$ Ethacrynic acid ( $1 \mathrm{mM})$ added to control incubation medium.

$\$$ Calculated by subtracting control value from that obtained when the medium contained $1 \mathrm{~mm}$ ethacrynic acid.

$\|$ Significantly different $(P<0.01)$ from mean value for patients J. A. and R. B. and from the mean value for normal subjects by $t$ test.

presence and absence of ethacrynic acid), and the effect of ethacrynic acid on sodium influx. On the basis of these differences, the patients with Bartter's syndrome have been divided into two groups which we have termed type I and type II.

In the six type I patients, erythrocyte sodium concentration was significantly greater than normal and fractional sodium outflux, both in the presence and absence of ethacrynic acid, was significantly less than normal. Addition of ethacrynic acid to the incubation medium produced an equivalent decrease in fractional sodium outflux in type I patients compared with normal. Sodium influx in these six patients did not differ significantly from normal; however, addition of ethacrynic acid produced a significant increase in sodium influx in the type I patients but produced a negligible decrease in sodium influx in normal subjects. In the two type II patients, in the patients with secondary hyperaldosteronism from other causes and in the patient with primary hyperaldosteronism, none of the measured parameters of erythrocyte sodium transport differed significantly from normal.

When erythrocytes from four type I patients (C. J., K. B., M. J., and D. C.) were incubated in a sodium-free medium, the average specific activity of the sodium leaving the cells $(3608 \pm 65 \mathrm{cpm} / \mathrm{nmole} \pm 1 \mathrm{sD})$ was not significantly different from the average specific activity

TABLE II

Absence of an Effect of Plasma on Fractional Sodium Outflux

\begin{tabular}{lcc}
\hline & \multicolumn{2}{c}{ Fractional Sodium outflux (hr-1) } \\
\cline { 2 - 3 } Subjects & Normal plasma & Bartter's plasma \\
\hline Bartter's syndrome & & \\
C. J. (19 M) & 0.396 & 0.382 \\
K. B. (4 F) & 0.340 & 0.346 \\
D. C. (13 F) & 0.352 & 0.351 \\
Normals & & \\
E. G. (20 M) & 0.487 & 0.493 \\
B. W. (18 F) & 0.526 & 0.515 \\
L. L. (9 F) & 0.493 & 0.486 \\
\hline
\end{tabular}

The values for all subjects were determined in duplicate. Age and sex of subjects in parentheses.

The sodium and potassium concentrations of the plasma were adjusted to 146 and $6 \mathrm{~mm}$ respectively.

Erythrocytes from each subject were preincubated for $3 \mathrm{hr}$ and incubated for $30 \mathrm{~min}$ in plasma from a normal subject and in plasma from a patient with Bartter's syndrome. 
in the cells $(3584 \pm 58)$. These data indicate that the source of the increased erythrocyte sodium concentration observed in these four patients was the decreased fractional sodium outflux. Garrahan and Glynn (13) have reported similar findings in erythrocytes from normal human subjects.

Table II illustrates that the decreased fractional sodium outflux in erythrocytes from three type I patients (C. J., K. B., and D. C.) persisted when the cells were preincubated and incubated in plasma from normal subjects. Similarly, plasma from these three patients did not alter fractional sodium outflux in erythrocytes from normal subjects.

Lactate production by erythrocytes from four type I patients (C. J., K. B., M. J., and J. E.) was $2.29 \pm 0.80$ mmoles/liter cells per $\mathrm{hr}$ (mean $\pm 1 \mathrm{SD}$ ) and was not significantly different from lactate production by erythrocytes from 10 normal subjects $(1.92 \pm 0.59)$.
Table III summarizes the results of studies of sodium transport in erythrocytes from relatives of three type I patients and of one type II patient. Erythrocyte sodium transport in the relatives of type I patients was significantly different from that in the relatives of the type II patient in terms of fractional sodium outflux (in both the presence and absence of ethacrynic acid) and the effect of ethacrynic acid on sodium influx, but not in terms of erythrocyte sodium concentration. Sodium transport in the relatives of type I patients differed significantly $(P<0.01)$ from that in normal subjects in that erythrocyte sodium concentration was increased, fractional sodium outflux was decreased both in the presence and absence of ethacrynic acid and ethacrynic acid stimulated sodium influx. In the relatives of the type II patient, fractional sodium outflux in the presence of ethacrynic acid and sodium influx were significantly less than normal $(P<0.01, P<0.05$ respectively $)$.

TABLE III

Sodium Transport in Erythrocytes from Relatives of Patients with Bartter's Syndrome

\begin{tabular}{|c|c|c|c|c|c|c|c|}
\hline \multirow[b]{2}{*}{ Subjects } & \multirow{2}{*}{$\begin{array}{c}\text { Erythrocyte } \\
\text { sodium } \\
\text { concentration }\end{array}$} & \multicolumn{3}{|c|}{ Fractional sodium outflux } & \multicolumn{3}{|c|}{ Sodium influx } \\
\hline & & Control* & +ETAt & Change§ & Control & +ETA & Change \\
\hline & $\begin{array}{l}\text { mmoles/ } \\
\text { liter cells }\end{array}$ & & $h r^{-1}$ & & & mmoles/liter cells & $h r$ \\
\hline \multicolumn{8}{|l|}{ C. J. family } \\
\hline Father (50) & 5.70 & 0.420 & 0.258 & -0.162 & 1.70 & 2.04 & 0.34 \\
\hline Mother (45) & 5.18 & 0.391 & 0.219 & -0.172 & 1.55 & 1.86 & 0.31 \\
\hline R. J. (3 F) & 7.29 & 0.346 & 0.156 & -0.190 & 1.56 & 1.58 & 0.02 \\
\hline D. J. $(14 \mathrm{~F})$ & 6.42 & 0.350 & 0.211 & -0.139 & 1.58 & 1.89 & 0.31 \\
\hline T. J. $(9 \mathrm{M})$ & 6.47 & 0.347 & 0.197 & -0.170 & 1.58 & 1.65 & 0.07 \\
\hline R. J. (16 M) & 7.38 & 0.372 & 0.219 & -0.153 & 1.57 & 1.83 & 0.26 \\
\hline \multicolumn{8}{|l|}{ K. B. family } \\
\hline Father (23) & 7.19 & 0.372 & 0.246 & -0.126 & 1.94 & 2.04 & 0.10 \\
\hline Mother (23) & 9.00 & 0.272 & 0.156 & -0.116 & 1.69 & 1.77 & 0.08 \\
\hline T. B. $(2 \mathrm{M})$ & 8.68 & 0.318 & 0.200 & -0.118 & 1.59 & 2.05 & 0.46 \\
\hline \multicolumn{8}{|l|}{ J. S. family } \\
\hline Father (38) & 7.38 & 0.430 & 0.276 & -0.154 & 1.98 & 2.11 & 0.13 \\
\hline Mother (26) & 5.28 & 0.419 & 0.303 & -0.116 & 1.67 & 1.70 & 0.03 \\
\hline Mean \pm 1 SD & $6.91 \pm 1.25$ & $0.37 \pm 0.05$ & $0.22 \pm 0.05$ & $-0.15 \pm 0.02$ & $1.67 \pm 0.15$ & $1.86 \pm 0.18$ & $0.19 \pm 0.15$ \\
\hline R. B. family & & & & & & & \\
\hline Father (42) & 6.60 & 0.487 & 0.268 & -0.219 & 1.86 & 1.61 & -0.25 \\
\hline Mother (34) & 5.52 & 0.468 & 0.271 & -0.197 & 1.20 & 1.28 & 0.08 \\
\hline S. B. $(5 \mathrm{M})$ & 5.37 & 0.447 & 0.308 & -0.139 & 1.40 & 1.44 & 0.04 \\
\hline J. B. $(6 \mathrm{~F})$ & 5.89 & 0.468 & 0.286 & -0.182 & 1.66 & 1.58 & -0.08 \\
\hline M. B. $(11 \mathrm{~F})$ & 5.38 & 0.448 & 0.268 & -0.180 & 1.39 & 1.34 & -0.05 \\
\hline Mean \pm 1 SD & $5.75 \pm 0.52$ & $0.46 \pm 0.02 \|$ & $0.28 \pm 0.02 \|$ & $-0.18 \pm 0.03$ & $1.50 \pm 0.26$ & $1.45 \pm 0.14 \|$ & $-0.05 \pm 0.13 \|$ \\
\hline
\end{tabular}

The values for all subjects were determined in duplicate.

Age and sex of subjects in parentheses.

* Control incubation medium (mM): $\mathrm{Na}, 146 ; \mathrm{K}, 6.0$.

$\ddagger$ Ethacrynic acid ( $1 \mathrm{~mm}$ ) added to control incubation medium.

$\S$ Calculated by subtracting control value from that obtained when the medium contained 1 mm ethacrynic acid.

\| Significantly different $(P<0.02)$ from mean value for all type I relatives. 
TABLE IV

Sodium Transport in Erythrocytes from Parents and Siblings of Patients with Bartter's Syndrome

\begin{tabular}{|c|c|c|c|c|c|c|c|}
\hline & \multirow{2}{*}{$\begin{array}{l}\text { Erythrocyte } \\
\text { sodium } \\
\text { concentration }\end{array}$} & \multicolumn{3}{|c|}{ Fractional sodium outflux } & \multicolumn{3}{|c|}{ Sodium influx } \\
\hline & & Control* & + ETA $\ddagger$ & Change§ & Control & +ETA & Change \\
\hline & $\begin{array}{l}\text { mmoles/ } \\
\text { liter cells }\end{array}$ & & $h r^{-1}$ & & & mmoles/liter cells & \\
\hline \multicolumn{8}{|l|}{ Siblings } \\
\hline Type I (5) & $7.25 \pm 0.92 \|$ & $0.35 \pm 0.02 \|$ & $0.20 \pm 0.02 \|$ & $-0.15 \pm 0.03$ & $1.58 \pm 0.01$ & $1.80 \pm 0.19 \|$ & $0.22 \pm 0.18$ \\
\hline Type II (3) & $5.55 \pm 0.30$ & $0.45 \pm 0.01$ & $0.29 \pm 0.02$ & $-0.17 \pm 0.02$ & $1.48 \pm 0.15$ & $1.45 \pm 0.12$ & $-0.03 \pm 0.06$ \\
\hline \multicolumn{8}{|l|}{ Parents } \\
\hline Type I (6) & $6.62 \pm 1.50$ & $0.38 \pm 0.06$ & $0.24 \pm 0.05$ & $-0.14 \pm 0.02 ף$ & $1.76 \pm 0.17$ & $1.92 \pm 0.17 \|$ & $0.16 \pm 0.12$ \\
\hline Type II (2) & $6.06 \pm 0.76$ & $0.48 \pm 0.01$ & $0.27 \pm 0.002$ & $-0.21 \pm 0.02$ & $1.53 \pm 0.47$ & $1.44 \pm 0.23$ & $-0.08 \pm 0.23$ \\
\hline
\end{tabular}

All values expressed as mean $\pm 1 \mathrm{SD}$.

* Control incubation medium (mM) : $\mathrm{Na}, 146 ; \mathrm{K}, 6.0$.

$\ddagger$ Ethacrynic acid (1 mM) added to control incubation medium.

\& Calculated by subtracting control value from that obtained when the medium contained 1 mm ethacrynic acid.

\| Significantly different $(P<0.02)$ from mean value for siblings of patient $\mathrm{R}$. B.

I Significantly different $(P<0.02)$ from mean value for parents of patient $\mathrm{R}$. B.

When each group of relatives was subdivided into siblings and parents (Table IV), sodium transport in erythrocytes from siblings of type I patients was significantly different from that in the siblings of the type II patient in terms of erythrocyte sodium concentration, fractional sodium outflux and sodium influx in the presence of ethacrynic acid. Sodium transport in erythrocytes from siblings of type I patients differed significantly $(P<0.01)$ from that in normal subjects in that erythrocyte sodium concentration was increased, fractional sodium outflux was decreased both in the presence and absence of ethacrynic acid, and ethacrynic acid stimulated sodium influx. In siblings of the type II patient, fractional sodium outflux in the presence of ethacrynic acid and sodium influx were decreased $(P<0.05)$ compared with normal subjects.

Sodium transport in erythrocytes from parents of type I patients was significantly different from that in the parents of the type II patient in terms of the decrease in fractional sodium outflux produced by adding ethacrynic acid to the medium and in terms of sodium influx in the presence of ethacrynic acid. Although these two groups did not differ significantly $(P<0.05)$ with respect to the mean control fractional outflux values, all of the values for the parents of the type I patient were lower than the values for the parents of the type II patient. Sodium transport in erythrocytes from parents of the type I patients differed significantly $(P<0.01)$ from that in normal subjects in that erythrocyte sodium concentration was increased, fractional sodium outflux was decreased both in the presence and absence of ethacrynic acid, and ethacrynic acid stimulated sodium influx. In the parents of the type II patient fractional sodium outflux in the presence of ethacrynic acid was below normal
$(P<0.05)$ and the decrease in fractional sodium outflux produced by adding ethacrynic acid to the medium was greater than normal $(P<0.05)$.

\section{DISCUSSION}

The altered erythrocyte sodium transport observed in the six type I patients cannot be attributed to hypokalemia or to elevated plasma concentrations of renin, angiotensin, or aldosterone since erythrocyte sodium transport was normal in the two type II patients with Bartter's syndrome and in the seven patients with secondary hyperaldosteronism from other causes. This conclusion is given additional support by previous studies from other laboratories $(4,14,15)$. The observations that plasma from type I patients had no significant effect on fractional sodium outflux in normal erythrocytes and that the altered sodium transport in erythrocytes from type I patients persisted in plasma from normal subjects indicate that the altered erythrocyte sodium transport in type I patients represents an intrinsic abnormality of their erythrocytes. We should mention, however, that these data do not exclude the rather unlikely existence in the type I patients of a circulating factor which interacts so slowly with erythrocytes that its effect can be neither reduced in erythrocytes from type I patients nor exerted on normal erythrocytes during the $3 \mathrm{hr}$ incubation period. The normal values for lactate production by erythrocytes from four of the type I patients exclude a defect in glycolysis as the source of the altered sodium transport. We are unable to explain the observation that ethacrynic acid increased sodium influx in the type I patients; however, this effect constitutes a qualitative difference whose understanding may 
help clarify the nature of the basic abnormality in these patients.

We are unable to reconcile the discrepancy between our findings and those of Gall, Vaitukaitis, Haddow, and Klein $(16,17)$ who reported a patient with Bartter's syndrome in whom erythrocyte sodium concentration, fractional sodium outflux, and sodium influx were increased. One possibility is that their patient (in whom juxtaglomerular hyperplasia was not demonstrated) had a syndrome whose basic etiology differed from that of the subjects used for our investigations. It is also possible that the discrepancy results from methodologic differences; however, the techniques used by Gall and coworkers are not described in sufficient detail to allow us to make a more precise statement. Finally, these authors' data for sodium transport in erythrocytes from a patient with Bartter's syndrome appear to be inconsistent. The $50 \%$ increase in sodium influx (which they suggest is the basic abnormality) is not of sufficient magnitude to account for an elevated erythrocyte sodium concentration when there is also an $80 \%$ increase in fractional sodium outflux. We were also unable to confirm these authors' observation of elevated fractional sodium outflux in erythrocytes from patients with primary and secondary hyperaldosteronism.

Others (9) have pointed out that with the exception of the cardinal features shared by all cases (juxtaglomerular hyperplasia, secondary hyperaldosteronism, hypokalemia, alkalosis, normal blood pressure, and absence of edema) there is marked variation in the physiologic and morphologic abnormalities in Bartter's syndrome. Our eight patients with Bartter's syndrome could be classified on the basis of their values for erythrocyte sodium transport: type I patients had increased erythrocyte sodium concentration and decreased fractional sodium outflux; type II patients had normal erythrocyte sodium concentration and fractional sodium outflux. We could find no correlation between the presence or absence of altered erythrocyte sodium transport and any of the other previously described physiologic or morphologic abnormalities in this clinical disorder $(1,3,6,9)$. Furthermore, the significance of classifying patients with Bartter's syndrome on the basis of their values for erythrocyte sodium transport must await clarification of the relation, if any, between altered erythrocyte sodium transport and the primary defect in this disorder.

Our finding of a similar alteration of sodium transport in six patients with Bartter's syndrome and in their relatives suggests that the defect in erythrocyte sodium transport is inherited. Although erythrocyte sodium concentration and fractional sodium outflux in R. B. and his relatives were normal, the patient's older sister (M. B.) has been previously demonstrated to have a diminished vasopressor response to angiotensin, an abnormally high aldosterone excretion while on a low sodium diet and a slight decrease in her serum potassium level (10). If the alterations of erythrocyte sodium transport are related to the etiologic abnormality in Bartter's syndrome, this disorder may be analogous to cystinuria where patients, all of whom have increased urinary cystine, lysine, and arginine, can be divided into three types depending on their values for the intestinal uptake of lysine, arginine, and cystine and values for urinary cystine excretion in their parents (18).

Our data are not sufficient to allow us to specify the mode of inheritance of the altered erythrocyte sodium transport in the type I patients and their relatives. We are also unable to relate the observed alterations in erythrocyte sodium transport to the primary defect in Bartter's syndrome; however, our data do suggest that the clinical features of this syndrome may be manifestations of an inherited defect in membrane cation transport.

\section{ACKNOWLEDGMENTS}

We thank Dr. Frederic Bartter for allowing us to study his patients and for reviewing this manuscript; Dr. Maria New and Dr. Michael Wald for referring their patients for study ; Dr. W. H. Wilkinson of Merck, Sharp \& Dohme for his gift of ethacrynic acid; Dr. David Alling for his assistance in statistical interpretation; and Mr. Edward Ginzler for his technical support.

\section{REFERENCES}

1. Bartter, F. C., P. Pronove, J. R. Gill, Jr., and R. C. MacCardle. 1962. Hyperplasia of the juxtaglomerular complex with hyperaldosteronism and hypokalemic alkalosis. Amer. J. Med. 33: 811.

2. Brackett, N. C., M. Koppel, R. E. Randall, Jr., and W. P. Nixon. 1968. Hyperplasia of the juxtaglomerular complex with secondary aldosteronism without hypertension (Bartter's syndrome). Amer. J. Med. 44: 803.

3. Cannon, P. J., J. M. Leeming, S. C. Sommers, R. Winters, and J. H. Laragh. 1968. Juxtaglomerular cell hyperplasia and secondary hyperaldosteronism (Bartter's syndrome) : a reevaluation of the pathophysiology. Medicine (Baltimore). 47: 107.

4. Trygstad, C. W., J. A. Mangos, J. M. B. Bloodworth, Jr., and C. C. Lobeck. 1969. A sibship with Bartter's syndrome: failure of total adrenalectomy to correct the potassium wasting. Pediatrics. 44: 234.

5. Visser, H. K. A., H. J. Degenhart, and E. Desmit. 1967. Mineralocorticoid excess in two brothers with dwarfism, hypokalemic alkalosis and normal blood pressure. Acta Endocrinol. 55: 661.

6. Sutherland, L. E., P. Hartroft, and J. U. Balis. 1970. Bartter's syndrome: a report of four cases, including three in one sibship with comparative evaluation of the juxtaglomerular apparatuses and glomeruli. Acta Pediat. Scand. Suppl. 201.

7. George, J. M., L. Wright, M. H. Bell, and F. C. Bartter. 1970. The syndrome of primary aldosteronism. Amer. J. Med. 48: 343 .

8. Conn, J. W., D. R. Rovner, and E. L. Cohen. 1965. Normal and altered function of the renin-angiotensinaldosterone system in man. Ann. Intern. Med. 63: 266. 
9. Wald, M. K., E. V. Perrin, and R. P. Bolande. 1971. Bartter's syndrome in early infancy. Pediatrics. 47: 254.

10. Greenberg, A. J., J. M. Arboit, M. I. New, and H. G. Worthen. 1966. Normotensive secondary hyperaldosteronism. J. Pediat. 69: 719.

11. Davidsohn, I., and B. B. Wells. 1963. Clinical Diagnosis by Laboratory Methods. W. B. Saunders Company, Philadelphia. 73.

12. Sachs, J. R., and L. G. Welt. 1967. The concentration dependence of active potassium transport in the human red blood cell. J. Clin. Invest. 46: 65.

13. Garrahan, P. J., and I. M. Glynn. 1967. The behavior of the sodium pump in red cells in the absence of external potassium. J. Physiol. (London). 192: 159.
14. Levin, M. L., F. C. Rector, Jr., and D. W. Seldin. 1968. Effects of potassium and ouabain on sodium transport in human red cells. Amer. J. Physiol. 214: 1328.

15. Glynn, I. M. 1957. The action of cardiac glycosides on sodium and potassium movements in human red cells. J. Physiol. (London). 136: 148.

16. Gall, G., P. Usher, J. C. Melby, and R. Klein. 1971. Effects of aldosterone and cortisol on human erythrocyte $\mathrm{Na}$ flux. J. Clin. Endocrinol. Metab. 32: 555.

17. Gall, G., J. Vaitukaitis, J. E. Haddow, and R. Klein. 1971. Erythrocyte $\mathrm{Na}$ flux in a patient with Bartter's syndrome. J. Clin. Endocrinol. Metab. 32: 562.

18. Rosenberg, L. E., S. Downing, J. L. Durant, and S. Segal. 1966. Cystinuria: biochemical evidence for three genetically distinct diseases. J. Clin. Invest. 45: 365 . 